\title{
Age-related outcomes following intracranial aneurysm treatment with the Pipeline Embolization Device: a subgroup analysis of the IntrePED registry
}

\author{
Waleed Brinjikji, MD, ${ }^{1}$ David F. Kallmes, MD, ${ }^{1,2}$ Harry J. Cloft, MD, PhD, ${ }^{1,2}$ and \\ Giuseppe Lanzino, MD ${ }^{1,2}$
}

Departments of ${ }^{1}$ Radiology and ${ }^{2}$ Neurosurgery, Mayo Clinic, Rochester, Minnesota

OBJECTIVE The association between age and outcomes following aneurysm treatment with flow diverters such as the Pipeline Embolization Device (PED) have not been well established. Using the International Retrospective Study of the Pipeline Embolization Device (IntrePED) registry, the authors assessed the age-related clinical outcomes of patients undergoing aneurysm embolization with the PED.

METHODS Patients with unruptured aneurysms in the IntrePED registry were divided into 4 age groups: $\leq 50,51-60$, $61-70$, and $>70$ years old. The rates of the following postoperative complications were compared between age groups using chi-square tests: spontaneous rupture, intracranial hemorrhage (ICH), ischemic stroke, parent artery stenosis, cranial neuropathy, neurological morbidity, neurological mortality, combined neurological morbidity and mortality, and all-cause mortality. The association between age and these complications was tested in a multivariate logistic regression analysis adjusted for sex, number of PEDs, and aneurysm size, location, and type.

RESULTS Seven hundred eleven patients with 820 unruptured aneurysms were included in this study. Univariate analysis demonstrated no significant difference in ICH rates across age groups (lowest $1.0 \%$ for patients $\leq 50$ years old and highest $5.0 \%$ for patients $>70$ years old, $p=0.097$ ). There was no difference in ischemic stroke rates (lowest $3.6 \%$ for patients $\leq 50$ years old and highest $6.0 \%$ for patients $50-60$ years old, $p=0.73$ ). Age $>70$ years old was associated with higher rates of neurological mortality; patients $>70$ years old had neurological mortality rates of $7.4 \%$ compared with $3.3 \%$ for patients $61-70$ years old, $2.7 \%$ for patients $51-60$ years old, and $0.5 \%$ for patients $\leq 50$ years old $(p=0.006$ ). On multivariate logistic regression analysis, increasing age was associated with higher odds of combined neurological morbidity and mortality (odds ratio 1.02, 95\% confidence interval 1.00-1.05; $p=0.03$ ).

CONCLUSIONS Increasing age is associated with higher neurological morbidity and mortality after Pipeline embolization of intracranial aneurysms. However, the overall complication rates of PED treatment in this group of highly selected elderly patients (> 70 years) were acceptably low, suggesting that age alone should not be considered an exclusion criterion when considering treatment of intracranial aneurysms with the PED.

http://thejns.org/doi/abs/10.3171/2015.5.JNS15327

KEY WORDS flow diverter; outcomes; age; aneurysm; vascular disorders

$\Lambda$ DVANCING age is a well-known risk factor for periprocedural morbidity and mortality after both surgery and coil embolization of intracranial aneurysms. ${ }^{4,11,13}$ Flow diversion is a more recently developed endovascular technique, and several single-center and multicenter series have reported encouraging early results, especially in the treatment of complex proximal internal carotid artery (ICA) aneurysms. ${ }^{1-3}$ However, given the relatively small size of these series, the effect of age on periprocedural complication rates and long-term clini- cal outcomes after flow diversion is difficult to establish. The International Retrospective Study of the Pipeline Embolization Device (IntrePED) is a large multicenter study conducted to assess the incidence of periprocedural complications after Pipeline (Medtronic) embolization of intracranial aneurysms in a "real world" scenario. ${ }^{8}$ This study provided us with a unique opportunity to assess the influence of advancing age on perioperative complications after flow diversion for intracranial aneurysms. In this study, we report age-related complications of IntrePED

ABBREVIATIONS $\mathrm{Cl}=$ confidence interval; ICA = internal carotid artery; ICH = intracranial hemorrhage; IntrePED = International Retrospective Study of the Pipeline Embolization Device; IPH = intraparenchymal hemorrhage; OR = odds ratio; PED = Pipeline Embolization Device; UCAS = Unruptured Cerebral Aneurysms Study. SUBMITTED February 10, 2015. ACCEPTED May 14, 2015.

INCLUDE WHEN CITING Published online November 6, 2015; DOI: 10.3171/2015.5.JNS15327. 
study patients receiving intracranial aneurysm treatment with the PED.

\section{Methods Study Patients}

Details regarding the inclusion and exclusion criteria, enrollment, and consent process for the IntrePED study are described in the original IntrePED study. ${ }^{8}$ In brief, we retrospectively evaluated all patients with unruptured intracranial aneurysms treated with the PED between July 2008 and February 2013 in 17 centers in 6 countries. This postmarket observational registry was funded and supported by Covidien with scientific oversight from study steering-committee members who were independent from the funding body. Seven hundred ninety-three patients treated for 906 aneurysms were enrolled, of whom 715 patients had 824 unruptured aneurysms, 76 patients had 76 ruptured aneurysms, and 6 aneurysms were of unknown status. Patients with ruptured aneurysms were excluded from this analysis. Four patients with unruptured aneurysms did not have age information available, thus 711 patients with 820 unruptured aneurysms were included in this study.

\section{Procedures and Data Collection}

As this was a retrospective study, procedural details and periprocedural patient management varied across centers. All centers used a common study protocol. Site investigators identified events of interest and all complications were reviewed in detail by an Adverse Events Review Committee, composed of 3 members of the Steering Committee, including the overall study principal investigator. The Adverse Events Review Committee was independent of the sponsor. A "major" adverse event was defined as an ongoing clinical deficit at 7 days following the event. The timing of all adverse events was recorded in relation to the timing of the PED procedure. A list of baseline characteristics collected for the purposes of the IntrePED study is provided in the original study. ${ }^{8}$

\section{Baseline Characteristics and Outcomes}

For the purposes of this study, patients were divided into 4 age groups by decade: $\leq 50,51-60,61-70$, and $>70$ years old. These age categories were arbitrarily defined by the investigators. The following baseline characteristics were compared between age groups: aneurysm location, aneurysm size, use of multiple PEDs, and mean procedure time. The rates of the following postoperative complications were compared between age groups: spontaneous rupture (including both subarachnoid hemorrhage and cavernous carotid fistula), intracranial hemorrhage (ICH), ischemic stroke, parent artery stenosis, cranial neuropathy, neurological mortality (death secondary to neurological causes such as stroke and ICH), neurological morbidity, combined neurological morbidity and mortality, and all-cause mortality. A "major" adverse event was defined as an ongoing clinical deficit at 7 days following the event.

\section{Statistical Analysis}

Statistical analyses were performed using SAS (version
9.2, SAS Institute Inc.). Descriptive statistics were used to present the data and to summarize the results. Discrete variables will be presented using frequency distributions and cross tabulations. Continuous variables will be summarized by presenting the number of observations $(\mathrm{N})$, mean, SD, median, and minimum and maximum values.

For categorical variables, differences between groups were tested using appropriate contingency table analyses (exact or chi-square approximations). For continuous variables, the differences were tested using the unpaired Student t-test or a nonparametric test, depending on variable distribution. Odds ratios (ORs) and 95\% confidence intervals (CIs) were obtained using multivariate logistic regression adjusted for sex, aneurysm size, aneurysm location, number of aneurysms treated, use of multiple PEDs, use of multiple exchange guidewires, practitioner's prior experience, and center volume. For the multivariate analysis, age was modeled as a continuous variable.

\section{Role of the Funding Source}

An academic principal investigator and an academic steering committee supervised trial design and operations. Both the principal investigator and steering committee were independent of the sponsor of the study (Covidien/Medtronic). The investigators wrote the report. The study sponsor was responsible for site management, data management, statistical analysis, and safety reporting. The corresponding author had full access to all study data and had final responsibility for the decision to submit for publication.

\section{Results \\ Patient Population and Baseline Characteristics}

In total, 711 patients with 820 unruptured aneurysms were included in this analysis (Table 1). The mean patient age was $57.7 \pm 13.8$ years. The median follow-up duration was 21 months. One hundred ninety-seven patients $(27.7 \%)$ were $\leq 50$ years old, $184(25.9 \%)$ were $51-60$ years old, 209 (29.4\%) were 61-70 years old, and 121 (17.0\%) were $>70$ years old. There were significant differences in the baseline characteristics between the 4 age groups in aneurysm location and size. Regarding aneurysm location-size criteria, patients $\leq 50$ years old were more likely to have ICA aneurysms $<10 \mathrm{~mm}$ in size (111 patients, $49.6 \%$ ) compared with patients $>70$ years old (34 patients, $24.5 \%$ ) and less likely to have posterior circulation aneurysms (23 patients, $10.3 \%$ ) when compared with patients $>$ 70 years old (22 patients, $15.8 \%$ ). Patients $>70$ years old had the largest mean aneurysm size $(13.5 \pm 7.6 \mathrm{~mm})$. Mean aneurysm size was $<12 \mathrm{~mm}$ for the other 3 age groups ( $\mathrm{p}$ $<0.0001)$. There was no difference in the number of patients receiving multiple PEDs between groups $(\mathrm{p}=0.14)$ or mean procedure time $(\mathrm{p}=0.36)$. These data are summarized in Table 1.

\section{Age-Related Outcomes}

Patients $>70$ years old had significantly higher rates of neurological mortality (9 patients, $7.4 \%$ ) when compared 
TABLE 1. Procedure and aneurysm measurements by age categories

\begin{tabular}{|c|c|c|c|c|c|}
\hline \multirow[b]{2}{*}{ Demographics } & \multicolumn{4}{|c|}{ Age (yrs) } & \multirow[b]{2}{*}{$p$ Value } \\
\hline & $\leq 50$ & $51-60$ & $61-70$ & $>70$ & \\
\hline No. of patients & 197 & 184 & 209 & 121 & \\
\hline No. of aneurysms & 224 & 216 & 241 & 139 & \\
\hline Aneurysm size (mm) & & & & & $<0.0001$ \\
\hline Mean \pm SD $(N)$ & $10.7 \pm 8.2(224)$ & $10.9 \pm 7.8(214)$ & $11.7 \pm 7.1(240)$ & $13.5 \pm 7.6(138)$ & \\
\hline Median (range) & $8.0(1.3-45.0)$ & $9.0(1.2-55.0)$ & $10.0(1.2-40.0)$ & $12.6(1.6-36.0)$ & \\
\hline Aneurysm location (\%) & & & & & 0.0014 \\
\hline $\mathrm{ICA} \geq 10 \mathrm{~mm}$ & $29.5(66 / 224)$ & $36.6(79 / 216)$ & $38.6(93 / 241)$ & $46.8(65 / 139)$ & \\
\hline ICA $<10 \mathrm{~mm}$ & $49.6(111 / 224)$ & $42.1(91 / 216)$ & $38.6(93 / 241)$ & $24.5(34 / 139)$ & \\
\hline Posterior (\%) & $10.3(23 / 224)$ & $9.7(21 / 216)$ & $9.1(22 / 241)$ & $15.8(22 / 139)$ & \\
\hline Other anterior (\%) & $9.8(22 / 224)$ & $11.1(24 / 216)$ & $13.7(33 / 241)$ & $10.8(15 / 139)$ & \\
\hline No. of PEDs (\%) & & & & & 0.1359 \\
\hline 1 & $69.8(155 / 222)$ & $59.3(128 / 216)$ & $63.9(154 / 241)$ & $66.2(92 / 139)$ & \\
\hline$>1$ & $30.2(67 / 222)$ & $40.7(88 / 216)$ & $36.1(87 / 241)$ & $33.8(47 / 139)$ & \\
\hline Procedure time (mins) & & & & & 0.3614 \\
\hline Mean \pm SD $(\mathrm{N})$ & $100.7 \pm 53.6(187)$ & $101.0 \pm 51.5(179)$ & $104.2 \pm 50.2(200)$ & $108.3 \pm 50.7(111)$ & \\
\hline Median (range) & $86.0(19.0-376.0)$ & $92.0(21.0-325.0)$ & $90.0(34.0-365.0)$ & $95.0(31.0-294.0)$ & \\
\hline
\end{tabular}

with patients $\leq 50$ years old ( 1 patient, $0.5 \%$ ), 51-60 years old (5 patients, 2.7\%), and 61-70 years old (7 patients, $3.3 \%$; $\mathrm{p}<0.01$ ). Patients $>70$ years old also had higher rates of all-cause mortality (10 patients, $8.3 \%)$ when compared with patients $\leq 50$ years old (1 patient, $0.5 \%$ ), 51-60 years old (7 patients, 3.8\%), and 61-70 years old (7 patients, 3.3\%; $\mathrm{p}<0.01$ ). There was no significant difference in total neurological morbidity $(\mathrm{p}=0.43)$ or combined neurological morbidity and mortality among the age groups $(\mathrm{p}=0.07)$.

Ischemic stroke rates were similar between age groups ( $p=0.73$ ) with rates of $3.6 \%$ (7 patients) for those $\leq 50$ years old, $6.0 \%$ (11 patients) for 51-60 years old, $4.3 \%$ (9 patients) for 61-70 years old, and $4.1 \%$ (5 patients) for $>70$ years old. The same was true for ICH as rates were 1.0\% (2 patients) for those patients $\leq 50$ years old, $1.1 \%$ ( 2 patients) for 51-60 years old, 1.9\% (4 patients) for 61-70 years old, and $5.0 \%$ (6 patients) for $>70$ years old $(\mathrm{p}=0.10)$. There were no differences in the rates of spontaneous rupture $(\mathrm{p}=1.0)$, or in-stent stenosis $(\mathrm{p}=0.17)$ between groups. These data are summarized in Table 2.

\section{Multivariate Logistic Regression Analysis}

When adjusting for sex, aneurysm size, aneurysm location, number of aneurysms treated, use of multiple PEDs, use of multiple exchange guidewires, practitioner's prior experience, and center volume, increasing age was associated with a higher odds of neurological mortality (OR $1.06,95 \%$ CI $1.02-1.10, \mathrm{p}<0.01$ ), combined neurological morbidity and mortality (OR 1.02, 95\% CI 1.00-1.05, p = 0.03 ), all-cause mortality (OR 1.06, 95\% CI 1.02-1.10, p $<0.01)$, and ICH (OR 1.05, 95\% CI 1.01-1.09, p = 0.024). These data are summarized in Table 3.

\section{Discussion}

Our study found that patients $>70$ years old are at high- er risk of neurological and all-cause mortality following intracranial aneurysm embolization with the PED but had similar rates of spontaneous rupture, $\mathrm{ICH}$, ischemic stroke, and neurological morbidity when compared with younger patients. The higher rate of neurological mortality in older patients was independent of the fact that older patients had significantly larger mean aneurysm size and were more likely to harbor posterior circulation aneurysms than their younger counterparts. Despite the higher rates of neurological and all-cause mortality found in older patients, the complication rates in these select elderly patients are acceptably low, suggesting that age alone should not be considered an exclusion criterion when considering PED treatment in patients of advanced age.

A number of prior studies have examined the association between advanced age and perioperative and longterm clinical outcomes following endovascular treatment of intracranial aneurysms with coiling. In a study of 355 patients undergoing elective treatment of unruptured aneurysms, Khosla et al. found that advanced age was associated with higher rates of major complications (17\% vs $7 \%)$ and higher rates of neurological disability $(8 \%$ vs $2 \%) .{ }^{9}$ One meta-analysis of endovascular treatment of intracranial aneurysms in 1511 elderly patients $(\geq 65$ years old) found that the rate of good neurological outcome without significant disability following unruptured aneurysm coil embolization was $82 \%$ at 6-12 months and $91 \%$ at 12 months or longer. ${ }^{11}$ The rates of combined disability and mortality during long-term follow-up after coil embolization of unruptured aneurysms was between $7 \%$ and $15 \%$, similar to our finding of $13 \% .^{11}$ Larger case series on endovascular coiling of unruptured aneurysms in the elderly in general report a rate of good neurological outcome of approximately $90 \%$.7.9 To our knowledge, the present series is the first to assess the association of age with complications related to this novel form of endovascular therapy. 
TABLE 2. Complications by age group

\begin{tabular}{lccccc}
\hline \multirow{2}{*}{ Complications } & \multicolumn{5}{c}{ Age $(\mathrm{yrs})^{*}$} \\
\cline { 2 - 5 } & $\leq 50(\mathrm{n}=197)$ & $51-60(\mathrm{n}=184)$ & $61-70(\mathrm{n}=209)$ & $>70(\mathrm{n}=121)$ & $\mathrm{p}$ Value \\
\hline Spontaneous rupture & $0.5(1)$ & $0.5(1)$ & $1.0(2)$ & $0.8(1)$ & 1.0000 \\
\hline ICH & $1.0(2)$ & $1.1(2)$ & $1.9(4)$ & $5.0(6)$ & 0.0966 \\
\hline Ischemic stroke & $3.6(7)$ & $6.0(11)$ & $4.3(9)$ & $4.1(5)$ & 0.7273 \\
\hline Parent artery stenosis & $1.0(2)$ & $0.0(0)$ & $0.0(0)$ & $0.0(0)$ & 0.1720 \\
\hline Cranial neuropathy & $0.0(0)$ & $0.0(0)$ & $0.0(0)$ & $1.7(2)$ & 0.0288 \\
\hline Neurological morbidity & $5.1(10)$ & $7.1(13)$ & $6.7(14)$ & $9.9(12)$ & 0.4256 \\
\hline Neurological mortality & $0.5(1)$ & $2.7(5)$ & $3.3(7)$ & $7.4(9)$ & 0.0062 \\
\hline Neurological morbidity \& mortality & $5.1(10)$ & $7.6(14)$ & $6.7(14)$ & $13.2(16)$ & 0.0734 \\
\hline All-cause mortality & $0.5(1)$ & $3.8(7)$ & $3.3(7)$ & $8.3(10)$ & 0.0029 \\
\hline
\end{tabular}

* Values given as percentage (no. of patients).

Although the rates of stroke and intraparenchymal hemorrhage (IPH) following PED treatment were similar between the elderly and younger cohorts, the rates of these complications in elderly patients are higher than those observed in endovascular coiling. Because of the thrombogenic nature of the bare metal construct of the PED, there is a high potential for thromboembolic events related to PED placement. It is likely for this reason that the rate of stroke in our elderly cohort is higher than that reported for elderly patients receiving endovascular coiling of unruptured aneurysms. However, the stroke rate found in our study is lower than the $11 \%$ stroke rate noted in the setting of stent-assisted coiling in the elderly population. ${ }^{10} \mathrm{PED}$ treatment has been associated with a higher rate of spontaneous IPH when compared with endovascular coiling. However, the exact etiology behind this disparity has yet to be determined. The spontaneous aneurysmal rupture rate following treatment was low in all groups.

While the complication rate in the elderly cohort was similar to that of the younger population, we did note higher rates of neurological and all-cause mortality in the elderly. It is possible that elderly patients who suffered perioperative complications of stroke or hemorrhage suffered higher morbidity and mortality as a result of these

TABLE 3. Multivariate analysis*

\begin{tabular}{lll}
\hline \multicolumn{1}{c}{ Complications } & OR $(95 \% \mathrm{Cl})$ & $\mathrm{p}$ Value \\
\hline Spontaneous rupture & $0.98(0.94-1.03)$ & 0.4635 \\
\hline $\mathrm{ICH}$ & $1.05(1.01-1.09)$ & 0.0238 \\
\hline Ischemic stroke & $1.0(0.98-1.03)$ & 0.7039 \\
\hline Parent artery stenosis & $0.79(0.59-1.06)$ & 0.1120 \\
\hline Cranial neuropathy & $1.02(0.97-1.07)$ & 0.3872 \\
\hline Neurological mortality & $1.06(1.02-1.10)$ & 0.0063 \\
\hline Neurological morbidity & $1.02(1.00-1.04)$ & 0.0987 \\
\hline Neurological morbidity \& mortality & $1.02(1.00-1.05)$ & 0.0322 \\
\hline All-cause mortality & $1.06(1.02-1.10)$ & 0.0030 \\
\hline
\end{tabular}

* For each of the complications, the OR was for increasing age. The analysis was adjusted for sex, aneurysm size, aneurysm location, number of aneurysms treated, use of multiple PEDs, use of multiple exchange guidewires, practitioner's prior experience, and center volume. complications when compared with their younger counterparts. In addition, elderly patients suffering severe ischemic or hemorrhagic events may have been more likely to receive withdrawal of care than their younger counterparts. Higher rates of all-cause mortality may be related to the general increase in comorbidities observed in patients with advancing age. Higher rates of cranial neuropathy may have been due to the fact that the aneurysms treated in the elderly group were generally larger.

It is important to point out that elderly patients receiving PED treatment in our study were highly selected. In general, elderly patients with significant comorbidities and poor life expectancy were not included in this registry and were deferred to more conservative management. It is also important to consider the relative morbidity and mortality rates observed in the elderly population in the context of large studies on the natural history of unruptured aneurysms. For example, while age was not found to be an independent risk factor for rupture in the Unruptured Cerebral Aneurysms Study (UCAS) of Japan, increasing aneurysm size was. The average size of the treated aneurysms in the elderly population in this study was $14 \mathrm{~mm}$, compared with 10-11 $\mathrm{mm}$ for the younger cohorts. In the UCAS, aneurysms $10-24 \mathrm{~mm}$ were associated with a rupture risk of $4.4 \%$ per year. Thus, the natural history of the treated unruptured aneurysms in the elderly cohort was not negligible.

Interestingly, our study found a trend toward higher instent stenosis rates among younger patients. Higher rates of in-stent stenosis in younger individuals have been reported in the stent-assisted coiling literature as well as within the coronary artery stenting literature. ${ }^{5,6,12}$ Higher rates of in-stent stenosis in younger individuals is believed to be due to a more exuberant reaction of the parent artery to the stent, resulting in higher rates of neointimal hyperplasia rather than factors such as lack of apposition or platelet aggregation and deposition at the site of the implant. ${ }^{5,6,12}$

\section{Limitations of the Study}

Our study has several limitations. The number of endpoint events was relatively low, and this limits the statistical power of subgroup analysis. The centers participating 
in this registry were selected based on their experience with the PED. Patients receiving treatment were a selected group, and some patients may not have been offered this treatment based on age-related considerations. Because this was a large, multinational registry, there was likely some variability in patient selection criteria from practitioner to practitioner and institution to institution. In general, PED candidates of all ages had no contraindications to dual antiplatelet therapy, were willing to be compliant with dual antiplatelet therapy, and were willing to be compliant with clinical and imaging follow-up evaluations. In general, elderly patients with significant comorbidities or poor life expectancy were not included in this registry and were directed to more conservative management. Nevertheless, this study provides useful data examining the relationship between age and periprocedural complications using this novel technology in a large number of patients and in a "real world" scenario distinct from the strict selection criteria of a prospective clinical trial.

\section{Conclusions}

This study found that highly selected elderly patients (> 70 years old) undergoing PED placement for treatment of unruptured intracranial aneurysms had similar rates of postprocedural morbidity, spontaneous rupture, IPH, and stroke when compared with their younger counterparts, but higher rates of neurological mortality. The overall complication rates of PED treatment in highly selected elderly patients were acceptably low, suggesting that age alone should not be considered an exclusion criteria when considering treatment of intracranial aneurysms with the PED.

\section{References}

1. Arrese I, Sarabia R, Pintado R, Delgado-Rodriguez M: Flow-diverter devices for intracranial aneurysms: systematic review and meta-analysis. Neurosurgery 73:193-200, 2013

2. Becske T, Kallmes DF, Saatci I, McDougall CG, Szikora I, Lanzino G, et al: Pipeline for uncoilable or failed aneurysms: results from a multicenter clinical trial. Radiology 267:858868,2013

3. Brinjikji W, Murad MH, Lanzino G, Cloft HJ, Kallmes DF: Endovascular treatment of intracranial aneurysms with flow diverters: a meta-analysis. Stroke 44:442-447, 2013

4. Brinjikji W, Rabinstein AA, Lanzino G, Kallmes DF, Cloft HJ: Effect of age on outcomes of treatment of unruptured cerebral aneurysms: a study of the National Inpatient Sample 2001-2008. Stroke 42:1320-1324, 2011

5. Du R, Zhang RY, Zhang Q, Shi YH, Hu J, Yang ZK, et al: Assessment of the relation between IVUS measurements and clinical outcome in elderly patients after sirolimus-eluting stent implantation for de novo coronary lesions. Int J Cardiovasc Imaging 28:1653-1662, 2012

6. Gao B, Safain MG, Malek AM: Enterprise stenting for intracranial aneurysm treatment induces dynamic and reversible age-dependent stenosis in cerebral arteries. J Neurointerv Surg 7:297-302, 2015

7. Gonzalez NR, Dusick JR, Duckwiler G, Tateshima S, Jahan R, Martin NA, et al: Endovascular coiling of intracranial aneurysms in elderly patients: report of 205 treated aneurysms. Neurosurgery 66:714-721, 2010

8. Kallmes DF, Hanel R, Lopes D, Boccardi E, Bonafe A, Cekirge $S$, et al: International retrospective study of the pipeline embolization device: a multicenter aneurysm treatment study. AJNR. Am J Neuroradiol 36:108-115, 2015

9. Khosla A, Brinjikji W, Cloft H, Lanzino G, Kallmes DF: Age-related complications following endovascular treatment of unruptured intracranial aneurysms. AJNR Am J Neuroradiol 33:953-957, 2012

10. Stiefel MF, Park MS, McDougall CG, Albuquerque FC: Endovascular treatment of unruptured intracranial aneurysms in the elderly: analysis of procedure related complications. J Neurointerv Surg 2:11-15, 2010

11. Sturiale CL, Brinjikji W, Murad MH, Lanzino G: Endovascular treatment of intracranial aneurysms in elderly patients: a systematic review and meta-analysis. Stroke 44:18971902, 2013

12. Turk AS, Levy EI, Albuquerque FC, Pride GL Jr, Woo H, Welch BG, et al: Influence of patient age and stenosis location on wingspan in-stent restenosis. AJNR Am J Neuroradiol 29:23-27, 2008

13. Wiebers DO, Whisnant JP, Huston J III, Meissner I, Brown RD Jr, Piepgras DG, et al : Unruptured intracranial aneurysms: natural history, clinical outcome, and risks of surgical and endovascular treatment. Lancet 362:103-110, 2003

\section{Disclosure}

This study was funded by Covidien/Medtronic. Dr. Kallmes has served as a consultant to Covidien/Medtronic and has received support for non-study-related clinical or research effort from Covidien/Medtronic, MicroVention, Codman, Sequent Medical, NeuroSigma, and Surmodics. Dr. Lanzino has served as a consultant to Covidien.

\section{Author Contributions}

Conception and design: all authors. Acquisition of data: Brinjikji, Kallmes, Lanzino. Analysis and interpretation of data: all authors. Drafting the article: Brinjikji, Kallmes, Lanzino. Critically revising the article: all authors. Reviewed submitted version of manuscript: all authors. Approved the final version of the manuscript on behalf of all authors: Brinjikji. Study supervision: Kallmes, Lanzino.

\section{Correspondence}

Waleed Brinjikji, Department of Radiology, Mayo Clinic, 200 First St. SW, OL1-112 SMH, Rochester, MN 55905. email: brinjikji.waleed@mayo.edu. 